\title{
On the explicit parametric equation of a general helix with first and second curvature in Nil 3-space
}

\author{
Şeyda Kılıçoğlu \\ Faculty of Education, Department of Elementary Mathematics Education, Baskent University, Ankara, Turkey
}

\section{Email address:}

seyda@baskent.edu.tr

\section{To cite this article:}

Şeyda Kılıçoğlu. On the Explicit Parametric Equation of a General Helix with First and Second Curvature in Nil 3-Space. Pure and Applied Mathematics Journal. Special Issue: Applications of Geometry. Vol. 4, No. 1-2, 2015, pp. 19-23. doi: 10.11648/j.pamj.s.2015040102.15

\begin{abstract}
Nil geometry is one of the eight geometries of Thurston's conjecture. In this paper we study in Nil 3-space and the Nil metric with respect to the standard coordinates $(x, y, z)$ is $g_{\mathrm{Nil}_{3}}=(d x)^{2}+(d y)^{2}+(d z-x d y)^{2}$ in $I^{3}$. In this paper, we find out the explicit parametric equation of a general helix. Further, we write the explicit equations Frenet vector fields, the first and the second curvatures of general helix in Nil 3-Space. The parametric equation the Normal and Binormal ruled surface of general helix in Nil 3-space in terms of their curvature and torsion has been already examined in [12], in Nil 3-Space.
\end{abstract}

Keywords: Nil Space, Helix, Curvatures

\section{Introduction}

In mathematics, Thurston's conjecture proposed a complete characterization of geometric structures on three-dimensional manifolds. The conjecture was proposed by William Thurston (1982), and implies several other conjectures, such as the Poincaré conjecture and Thurston's elliptization conjecture. Thurston's geometrization conjecture states that; Certain three-dimensional topological spaces each have a unique geometric structure that can be associated with them. It is an analogue of the uniformization theorem for two-dimensional surfaces, which states that every simply-connected Riemann surface can be given one of three geometries (Euclidean, spherical, or hyperbolic). In three dimensions, it is not always possible to assign a single geometry to a whole topological space. Instead, the geometrization conjecture states that every closed 3-manifold can be decomposed in a canonical way into pieces that each have one of eight types of geometric structure. Thurston's conjecture is that, after you split a three-manifold into its connected sum and the Jaco-Shalen-Johannson torus decomposition, the remaining components each admit exactly one of the following geometries

Euclidean geometry,

Hyperbolic geometry,

Spherical geometry,

The geometry of $\mathrm{S} 2 \times \mathrm{R}$,

The geometry of $\mathrm{H} 2 \times \mathrm{R}$,

The geometry of the universal cover SL_2R of the Lie group SL_2R,

Nil geometry,

Sol geometry.

For more detail see [13].

A nilmanifold is a differentiable manifold which has a transitive nilpotent group of diffeomorphisms acting on it. In the Riemannian category, there is also a good notion of a nilmanifold. A Riemannian manifold is called a homogeneous nilmanifold if there exist a nilpotent group of isometries acting transitively on it. The requirement that the transitive nilpotent group acts by isometries leads to the following rigid characterization: every homogeneous nilmanifold is isometric to a nilpotent Lie group with left-invariant metric (see [4]).

The two-parameter family of metrics first appeared in the works of Bianchi, Cartan and Vranceanu, these spaces are often referred to as Bianchi-Cartan-Vranceanu spaces, or BCV-spaces for short. Some well-known examples of BCV-spaces are the Riemannian product spaces $\mathrm{S}^{2} \times \mathrm{R}, \mathrm{H}^{2} \times \mathrm{R}$ and the 3-dimensional Heisenberg group [5]. Let $\kappa$ and $\tau$ be real numbers, with $\tau \geq 0$. The Bianchi-Cartan-Vranceanu spaces, (BCV-spaces) $\mathrm{M}^{3}(\kappa, \tau)$ is defined as the set

$$
\left\{(\mathrm{x}, \mathrm{y}, \mathrm{z}) \in \mathrm{IR}^{3}: 1+(\kappa / 4)\left(\mathrm{x}^{2}+\mathrm{y}^{2}\right)>0\right\}
$$

equipped with metric

$$
\begin{gathered}
\mathrm{ds}^{2}=\left(\left(\mathrm{dx}^{2}+\mathrm{dy}^{2}\right) /\left(\left(1+(\kappa / 4)\left(\mathrm{x}^{2}+\mathrm{y}^{2}\right)\right)^{2}\right)\right) \\
+\left(\mathrm{dz}+\tau\left((\mathrm{ydx}-\mathrm{xdy}) /\left(1+(\kappa / 4)\left(\mathrm{x}^{2}+\mathrm{y}^{2}\right)\right)\right)\right)^{2} .
\end{gathered}
$$




$$
\begin{gathered}
* \text { if } \kappa=\tau=0, \text { then } \mathrm{M}^{3}(\kappa, \tau) \cong \mathrm{IE}^{3} \\
* \text { if } \kappa=0 \text { and } \tau \neq 0, \text { then } \mathrm{M}^{3}(\kappa, \tau) \cong \mathrm{Nil}_{3} .
\end{gathered}
$$

More details can be found in [4] and [1].

In [5], it is restricted to the 3-dimensional Heisenberg group coming from $\mathrm{R}^{2}$ with the canonical symplectic form $\omega\left((\mathrm{x}, \mathrm{y}),\left(\mathrm{x}_{1}, \mathrm{y}_{1}\right)\right)=\mathrm{xy}_{1}-\mathrm{x}_{1} \mathrm{y}$, i.e., they consider $\mathrm{R}^{3}$ with the group operation

$$
(\mathrm{x}, \mathrm{y}, \mathrm{z}) *\left(\mathrm{x}_{1}, \mathrm{y}_{1}, \mathrm{z}_{1}\right)=\left(\mathrm{x}+\mathrm{x}_{1}, \mathrm{y}+\mathrm{y}_{1}, \mathrm{z}+\mathrm{z}_{1}+\left(\left(\mathrm{xy}_{1}\right) / 2\right)-\left(\left(\mathrm{x}_{1} \mathrm{y}\right) / 2\right)\right) .
$$

For every non-zero number $\tau$ the following Riemannian metric on $\left(\mathrm{R}^{3}, *\right)$ is left invariant:

$$
\mathrm{ds}^{2}=\mathrm{dx}^{2}+\mathrm{dy}^{2}+4 \tau^{2}(\mathrm{dz}+((\mathrm{ydx}-\mathrm{xdy}) / 2))^{2} .
$$

After the change of coordinates $(x, y, 2 \tau z) \rightarrow(x, y, z)$, this metric is expressed as

$$
\mathrm{ds}^{2}=\mathrm{dx}^{2}+\mathrm{dy}^{2}+(\mathrm{dz}+\tau(\mathrm{ydx}-\mathrm{xdy}))^{2} .
$$

By some authors the notation Nil 3-space is only used if $\tau=(1 / 2)$. We will use the notation $\mathrm{Nil}_{3}$ in short. It is well known that Nil space is isometric to Heisenberg space. The geometry of Nil is the three dimensional Lie group of all real 3 triangular matrices of the form

$$
\left[\begin{array}{lll}
1 & x & z \\
0 & 1 & y \\
0 & 0 & 1
\end{array}\right] .
$$

Let $\left(\mathrm{IR}^{3}, \mathrm{~g}_{\mathrm{Ni}_{3}}\right)$ denote Nil space, where the metric with respect to the standard coordinates $(\mathrm{x}, \mathrm{y}, \mathrm{z})$ in $\mathrm{IR}^{3}$ can be written [14] as

$$
\mathrm{g}_{\mathrm{Nil}_{3}}=(\mathrm{dx})^{2}+(\mathrm{dy})^{2}+(\mathrm{dz}-\mathrm{xdy})^{2} .
$$

Hence we get the symetric tensor field $\mathrm{g}_{\mathrm{Nil}_{3}}$ on $\mathrm{Nil}_{3}$ by components.

$$
\mathrm{g}_{\mathrm{ij}}=\left[\begin{array}{ccc}
1 & 0 & 0 \\
0 & 1+x^{2} & -x \\
0 & -x & 1
\end{array}\right]
$$

Note that the Nil metric can also be written as:

$$
\mathrm{ds}^{2}=\sum_{\mathrm{i}=1}^{3} \omega \mathrm{i} \otimes \omega \mathrm{i},
$$

where $\omega^{1}=\mathrm{dx}, \omega^{2}=\mathrm{dy}, \omega^{3}=\mathrm{dz}-\mathrm{xdy}$, and the orthonormal basis dual to the 1 -forms is

$$
\mathrm{E}_{1}=(\partial /(\partial \mathrm{x})), \mathrm{E}_{2}=(\partial /(\partial \mathrm{y}))+\mathrm{x}(\partial /(\partial \mathrm{z})), \mathrm{E}_{3}=(\partial /(\partial \mathrm{z})) .
$$

With respect to this orthonormal basis, the Levi-Civita connection and the Lie brackets can be easily computed as:

$$
\begin{aligned}
& \nabla_{\mathrm{E}_{1}} \mathrm{E}_{1}=0, \quad \nabla_{\mathrm{E}_{1}} \mathrm{E}_{2}=(1 / 2) \mathrm{E}_{3}, \quad \nabla_{\mathrm{E}_{1}} \mathrm{E}_{3}=((-1) / 2) \mathrm{E}_{2} \\
& \nabla_{\mathrm{E}_{2}} \mathrm{E}_{1}=((-1) / 2) \mathrm{E}_{3}, \quad \nabla_{\mathrm{E}_{2}} \mathrm{E}_{2}=0, \quad \nabla_{\mathrm{E}_{2}} \mathrm{E}_{3}=(1 / 2) \mathrm{E}_{1} \\
& \nabla_{\mathrm{E}_{3}} \mathrm{E}_{1}=((-1) / 2) \mathrm{E}_{2}, \quad \nabla_{\mathrm{E}_{3}} \mathrm{E}_{2}=(1 / 2) \mathrm{E}_{1}, \quad \nabla_{\mathrm{E}_{3}} \mathrm{E}_{3}=0 \text {. }
\end{aligned}
$$

$$
\left[\mathrm{E}_{1}, \mathrm{E}_{2}\right]=\mathrm{E}_{3}, \quad\left[\mathrm{E}_{2}, \mathrm{E}_{3}\right]=0, \quad\left[\mathrm{E}_{1}, \mathrm{E}_{3}\right]=0 .
$$

Hence

$$
\left[\begin{array}{ccc}
0 & \frac{1}{2} E_{3} & \frac{-1}{2} E_{2} \\
\frac{-1}{2} E_{3} & 0 & \frac{1}{2} E_{1} \\
\frac{-1}{2} E_{2} & \frac{1}{2} E_{1} & 0
\end{array}\right]
$$

is the matrix with (i,j)- element in the table equals $\nabla_{\mathrm{Ei}} \mathrm{E}_{\mathrm{j}}$ for the basis $\left\{\mathrm{E}_{1}, \mathrm{E}_{2}, \mathrm{E}_{3}\right\}$. See for more details [14].

\section{The Parametric Equation of General Helix in Nil 3-Space}

\subsection{Riemannian Structure of Nil Space}

Helix is one of the fascinating curve in science and nature. In this section, we study on the general helices in the $\mathrm{Nil}_{3}$. We characterize the general helices in terms of their curvature and torsion. A curve of constant slope or general helix is defined by the property that the tangent makes a constant angle with a fixed straight line (the axis of the helix). A classical result stated by M. A. Lancret in 1802 and first proved by B. de Saint Venant in 1845 (see [7] and [2] for details) is: A necessary and sufficient condition that a curve be a helix is that the ratio of curvature to torsion be constant. Helices are examined in [9] and [6]. Let $\alpha$ be a helix that lies on the cylinder. A helix which lies on the cylinder is called cylindrical helix or general helix. Assume that $\{\mathrm{T}, \mathrm{N}, \mathrm{B}, \kappa, \tau\}$ be the Frenet apparatus along the curve $\alpha$. It has been known that the curve $\alpha$ is a cylindrical helix if and only if $((\kappa / \tau))$ is constant, then $((\kappa / \tau))^{\prime}=0$ where $\kappa$ and $\tau$ are the curvatures of $\alpha$. If the curve is a general helix, the ratio of the first curvature of the curve to the torsion of the curve must be constant. We call a curve a circular helix if both $\tau \neq 0$ and $\kappa$ are constant. Then, the Frenet frame satisfies the following Frenet-Serret equations

$$
\begin{aligned}
& \nabla_{\mathrm{T}} \mathrm{T}=\kappa \mathrm{N}, \\
& \nabla_{\mathrm{T}} \mathrm{N}=-\kappa \mathrm{T}+\tau \mathrm{B}, \\
& \nabla_{\mathrm{T}} \mathrm{B}=-\tau \mathrm{N} .
\end{aligned}
$$

With respect to the orthonormal basis $\left\{\mathrm{E}_{1}, \mathrm{E}_{2}, \mathrm{E}_{3}\right\}$, we can write

$$
\begin{aligned}
& T=T_{1} E_{1}+T_{2} E_{2}+T_{3} E_{3}, \\
& N=N_{1} E_{1}+N_{2} E_{2}+N_{3} E_{3}, \\
& B=T \times N=B_{1} E_{1}+B_{2} E_{2}+B_{3} E_{3} .
\end{aligned}
$$

Parametric equations of general helices in the sol space $\mathrm{Sol}_{3}$ are examined in [3]. Normal ruled surfaces of general helices in the Sol space $\mathrm{Sol}_{3}$ are examined in [8].

Normal and Binormal ruled surfaces of general helices in Nil 3-space with the Riemannian Structure of Nil 3-space are examined in [12].

Parametric equation of general helix and all the Frenet 
apparatus are examined as in the following theorems.

\subsection{The parametric equation of General Helices in Nil Space $\mathrm{Nil}_{3}$}

\section{Theorem:}

Let $\alpha: \mathrm{I} \rightarrow \mathrm{Nil}_{3}$ be a unit speed non-geodesic general helix. Then, the equation of a unit speed non-geodesic general helix $\alpha$, with respect to the orthonormal basis,

$\left\{\mathrm{E}_{1}, \mathrm{E}_{2}, \mathrm{E}_{3}\right\}$,

$$
\begin{aligned}
\alpha(\mathrm{s}) & \left.=\left((\sin \beta) /\left(\mathrm{C}_{1}\right)\right) \sin \mathrm{D}+\mathrm{C}_{3}\right) \mathrm{E}_{1}+\left(\left((-\sin \beta) /\left(\mathrm{C}_{1}\right)\right) \cos \mathrm{D}+\mathrm{C}_{4}\right) \mathrm{E}_{2} \\
& +\left(\left(\left(\sin ^{2} \beta\right) /\left(4 \mathrm{C}_{1}{ }^{2}\right)\right) \sin 2 \mathrm{D}-\left(\left(\mathrm{C}_{4} \sin \beta\right) /\left(\mathrm{C}_{1}\right)\right) \sin \mathrm{D}\right. \\
& \left.+\left(\left(\left(\sin ^{2} \beta\right) /\left(2 \mathrm{C}_{1}\right)\right)+\cos \beta\right) s-\mathrm{C}_{3} \mathrm{C}_{4}+\mathrm{C}_{5}\right) \mathrm{E}_{3},
\end{aligned}
$$

where we take $\mathrm{D}=\mathrm{C}_{1} \mathrm{~s}+\mathrm{C}_{2}, \mathrm{C}_{1}, \mathrm{C}_{2} \in \mathrm{IR}$.

Proof:

Assume that $\alpha: \mathrm{I} \rightarrow \mathrm{Nil}_{3}$ be a unit speed non-geodesic general helix. So, without loss of generality, we take its axis as parallel to the vector $\mathrm{E}_{3}$. Then

$$
\operatorname{gnil}_{3}\left(\mathrm{~T}, \mathrm{E}_{3}\right)=\mathrm{T}_{3}=\cos \beta \text {, }
$$

where $\beta$ is constant angle. On the other hand the tangent vector $\mathrm{T}$ is an unit vector, so the following condition is satisfied $\mathrm{T}_{1}{ }^{2}+\mathrm{T}_{2}{ }^{2}=1-\cos ^{2} \beta$. Since $\cos ^{2} \beta+\sin ^{2} \beta=1$, we have the general solution of $\mathrm{T}_{1}{ }^{2}+\mathrm{T}_{2}{ }^{2}=\sin ^{2} \beta$ can be written in the following form

$$
\begin{aligned}
& \mathrm{T}_{1}=\sin \beta \cos \mathrm{D}, \\
& \mathrm{T}_{2}=\sin \beta \sin \mathrm{D}, \\
& \mathrm{T}_{3}=\cos \beta
\end{aligned}
$$

Also, without loss of generality, where we take $\mathrm{D}=\mathrm{C}_{1} \mathrm{~s}+\mathrm{C}_{2}$ where $C_{1}, C_{2} \in I R$. So, substituting the components $T_{1}, T_{2}$ and $\mathrm{T}_{3}$ in the equation, we have the following equation

$$
\mathrm{T}=\sin \beta \cos \mathrm{DE}_{1}+\sin \beta \sin \mathrm{DE}_{2}+\cos \beta \mathrm{E}_{3} .
$$

Definition of the tangent vector field T, give us;

$$
\begin{gathered}
((d x) /(d s))=\sin \beta \cos D \\
((d y) /(d s))=\sin \beta \sin D \\
((d z) /(d s))=x \sin \beta \sin D+\cos \beta .
\end{gathered}
$$

Integrating both sides, we have

$$
\begin{gathered}
\Rightarrow \mathrm{x}(\mathrm{s})=\left((\sin \beta) /\left(\mathrm{C}_{1}\right)\right) \sin \mathrm{D}+\mathrm{C}_{3} \\
\Rightarrow \mathrm{y}(\mathrm{s})=\left((-\sin \beta) /\left(\mathrm{C}_{1}\right)\right) \cos \mathrm{D}+\mathrm{C}_{4} \\
\Rightarrow \mathrm{z}(\mathrm{s})=\left(-\left(\left(\sin ^{2} \beta\right) /\left(4 \mathrm{C}_{1}{ }^{2}\right)\right) \sin 2 \mathrm{D}\right. \\
\quad-\left(\left(\mathrm{C}_{3} \sin \beta\right) /\left(\mathrm{C}_{1}\right)\right) \cos \mathrm{D} \\
\left.\quad+\left(\left(\left(\sin ^{2} \beta\right) /\left(2 \mathrm{C}_{1}\right)\right)+\cos \beta\right) \mathrm{s}\right)+\mathrm{C}_{5}
\end{gathered}
$$

where $\mathrm{C}_{3}, \mathrm{C}_{4}, \mathrm{C}_{5}$ are constant of integration. Substituting all them in $\alpha(\mathrm{s})$, this proves our assertion. Thus, the proof of theorem is completed.

\section{The Curvatures of the General Helix in Nil 3-Space}

\subsection{First Curvature of the General Helix in Nil Space $\mathrm{Nil}_{3}$}

Theorem:

The first curvature (curvature ) of the general helix in Nil Space $\mathrm{Nil}_{3}$ is

$$
\kappa=\left(\cos \beta-C_{1}\right) \sin \beta, \quad\left(\cos \beta-C_{1}\right) \sin \beta>0
$$

Proof:

Assume that $\alpha: \mathrm{I} \rightarrow \mathrm{Nil}_{3}$ be a unit speed non-geodesic general helix with

$$
\mathrm{T}=\sin \beta \cos \mathrm{DE}_{1}+\sin \beta \sin \mathrm{DE}_{2}+\cos \beta \mathrm{E}_{3} .
$$

The Levi-Civita connection and Lie brackets can be easily computed as:

$$
\begin{aligned}
\nabla_{\mathrm{T}} \mathrm{T} & =\mathrm{T}_{1}^{\prime} \mathrm{E}_{1}-(1 / 2) \mathrm{T}_{1} \mathrm{~T}_{2} \mathrm{E}_{3}+((-1) / 2) \mathrm{T}_{1} \mathrm{~T}_{3} \mathrm{E}_{2} \\
& +\mathrm{T}_{2}^{\prime} \mathrm{E}_{2}+(1 / 2) \mathrm{T}_{2} \mathrm{~T}_{1} \mathrm{E}_{3}+(1 / 2) \mathrm{T}_{2} \mathrm{~T}_{3} \mathrm{E}_{1} \\
& +\mathrm{T}_{3}^{\prime} \mathrm{E}_{3}-(1 / 2) \mathrm{T}_{3} \mathrm{~T}_{1} \mathrm{E}_{2}+(1 / 2) \mathrm{T}_{3} \mathrm{~T}_{2} \mathrm{E}_{1} \\
= & \left(\mathrm{T}_{1}^{\prime}+\mathrm{T}_{2} \mathrm{~T}_{3}\right) \mathrm{E}_{1}+\left(\mathrm{T}_{2}^{\prime}-\mathrm{T}_{1} \mathrm{~T}_{3}\right) \mathrm{E}_{2}+\left(\mathrm{T}_{3}^{\prime}\right) \mathrm{E}_{3}
\end{aligned}
$$

By substituting $T_{1}, T_{2}, T_{3}$ and derivates, we get

$$
\begin{aligned}
\nabla_{\mathrm{T}} \mathrm{T} & =\left(\cos \beta-\mathrm{C}_{1}\right)\left(\sin \beta \sin \mathrm{DE}_{1}-\sin \beta \cos \mathrm{DE}_{2}\right) \\
& =\left(\cos \beta-\mathrm{C}_{1}\right) \sin \beta\left(\sin \mathrm{DE}_{1}-\cos \mathrm{DE}_{2}\right) .
\end{aligned}
$$

Since $\kappa=\mathrm{g}_{\mathrm{Nil}}\left(\nabla_{\mathrm{T}} \mathrm{T}, \mathrm{N}\right)$ and $\mathrm{N}=(1 / \kappa) \nabla_{\mathrm{T}} \mathrm{T}$ we have

$$
\begin{gathered}
\kappa=\mathrm{g}_{\mathrm{Nil3}}\left(\nabla_{\mathrm{T}} \mathrm{T},(1 / \kappa) \nabla_{\mathrm{T}} \mathrm{T}\right) \kappa^{2}=\mathrm{gNil} 3\left(\nabla_{\mathrm{T}} \mathrm{T}, \nabla_{\mathrm{T}} \mathrm{T}\right) \\
\kappa^{2}=\left(\mathrm{C}_{1} \sin \beta-\sin \beta \cos \beta\right)^{2} \\
=\left(\cos \beta-\mathrm{C}_{1}\right)^{2} \sin ^{2} \beta
\end{gathered}
$$

and also for $\left(\cos \beta-C_{1}\right) \sin \beta>0$, first curvature is

$$
\kappa=\left(C_{1}-\cos \beta\right) \sin \beta \text {. }
$$

\subsubsection{The Normal Vector Fields of the General Helix}

The following theorem gives us the explicit parametric equation of normal vector fields in $\mathrm{Nil}_{3}$.

Theorem:

Let $\alpha: \mathrm{I} \rightarrow \mathrm{Nil}_{3}$ be a unit speed non-geodesic general helix. Then, the normal vector field of the general helix is

$$
\mathrm{N}=(\sin \mathrm{D},-\cos \mathrm{D}, 0)
$$

where we take $\mathrm{D}=\mathrm{C}_{1} \mathrm{~s}+\mathrm{C}_{2}, \mathrm{C}_{1}, \mathrm{C}_{2} \in \mathrm{IR}$.

Proof:

Let $\alpha: \mathrm{I} \rightarrow \mathrm{Nil}_{3}$ be a unit speed non-geodesic general helix. By the use of Frenet formula $\nabla_{\mathrm{T}} \mathrm{T}=\kappa \mathrm{N}$,

$$
\begin{aligned}
\kappa \mathrm{N} & =\left(\cos \beta-\mathrm{C}_{1}\right)\left(\sin \beta \sin \mathrm{DE}_{1}-\sin \beta \cos \mathrm{DE}_{2}\right) \\
& =\left(\cos \beta-\mathrm{C}_{1}\right) \sin \beta\left(\sin \mathrm{DE}_{1}-\cos \mathrm{DE}_{2}\right) .
\end{aligned}
$$

Hence the normal vector field of the general helix is 


$$
\begin{aligned}
\mathrm{N}= & (1 / \kappa)\left(\left(-\mathrm{C}_{1} \sin \beta+((\sin 2 \beta) / 2)\right) \sin _{1} \mathrm{E}_{1}\right. \\
& \left.+\left(\mathrm{C}_{1} \sin \beta-((\sin 2 \beta) / 2)\right) \cos \mathrm{DE}_{2}\right)
\end{aligned}
$$

where we take $\mathrm{D}=\mathrm{C}_{1} \mathrm{~s}+\mathrm{C}_{2}$, where $\mathrm{C}_{1}, \mathrm{C}_{2} \in \mathrm{IR}$. Also we know that; $\kappa=\left(\mathrm{C}_{1}-\cos \beta\right) \sin \beta$, so

$$
\mathrm{N}=(1 / \kappa) \nabla_{\mathrm{T}} \mathrm{T}
$$

$\left.\mathrm{N}=1 /\left(\sin \beta\left(\cos \beta-\mathrm{C}_{1}\right)\right)\right)\left(\cos \beta-\mathrm{C}_{1}\right)\left(\sin \beta \sin \mathrm{DE}_{1}-\sin \beta \cos \mathrm{DE}_{2}\right)$

$=\sin \mathrm{DE}_{1}-\cos \mathrm{DE}_{2}$

$\mathrm{N}=(\sin \mathrm{D},-\cos \mathrm{D}, 0)$

or substituting

$$
\mathrm{E}_{1}=(\partial /(\partial \mathrm{x})), \mathrm{E}_{2}=(\partial /(\partial \mathrm{y}))+\mathrm{x}(\partial /(\partial \mathrm{z})), \mathrm{E}_{3}=(\partial /(\partial \mathrm{z}))
$$

In

$$
\begin{aligned}
& \mathrm{N}=\operatorname{sinDE}_{1}-\cos _{2} \mathrm{E}_{2} \\
& \mathrm{~N}=\sin \mathrm{D}(\partial /(\partial \mathrm{x}))-\cos \mathrm{D}((\partial /(\partial \mathrm{y}))+\mathrm{x}(\partial /(\partial \mathrm{z})))
\end{aligned}
$$

$\mathrm{N}=\sin \mathrm{D}(\partial /(\partial \mathrm{x}))-\cos \mathrm{D}(\partial /(\partial \mathrm{y}))-\left(\left((\sin \beta) /\left(\mathrm{C}_{1}\right)\right) \sin \mathrm{D}-\mathrm{C}_{3}\right) \cos \mathrm{D}(\partial /($ $\partial \mathrm{z})$ ).

\subsection{Second Curvature (Torsion) of the General Helix in Nil Space $\mathrm{Nil}_{3}$}

Theorem:

The second curvature (torsion) of the general helix in Nil Space $\mathrm{Nil}_{3}$ is

$$
\tau=\left(\mathrm{C}_{1}{ }^{2}-\mathrm{C}_{1} \cos \beta+(1 / 4)\right)^{1 / 2}
$$

Proof:

With the Levi-Civita connection and Lie brackets can be easily computed as:

$$
\begin{aligned}
\nabla_{\mathrm{T}} \mathrm{N}= & \left(\mathrm{N}_{1}^{\prime}+(1 / 2) \mathrm{N}_{2} \mathrm{~T}_{3}+(1 / 2) \mathrm{N}_{3} \mathrm{~T}_{2}\right) \mathrm{E}_{1} \\
& +\left(\mathrm{N}_{2}^{\prime}+((-1) / 2) \mathrm{N}_{1} \mathrm{~T}_{3}+((-1) / 2) \mathrm{N}_{3} \mathrm{~T}_{1}\right) \mathrm{E}_{2} \\
& +\left(\mathrm{N}_{3}^{\prime}+(1 / 2) \mathrm{N}_{2} \mathrm{~T}_{1}+((-1) / 2) \mathrm{N}_{1} \mathrm{~T}_{2}\right) \mathrm{E}_{3} .
\end{aligned}
$$

Also for $\mathrm{N}=\sin \mathrm{D} \mathrm{E}_{1}-\cos \mathrm{D} \mathrm{E}_{2}$, we know that

$$
\begin{aligned}
& \mathrm{N}_{1}=\sin \mathrm{D} ; \mathrm{N}_{1}^{\prime}=\mathrm{C}_{1} \cos \mathrm{D} \\
& \mathrm{N}_{2}=-\cos \mathrm{D} ; \mathrm{N}_{2}^{\prime}=\mathrm{C}_{1} \sin \mathrm{D} \\
& \mathrm{N}_{3}=0, \mathrm{~N}_{3}^{\prime}=0 .
\end{aligned}
$$

Now it is easy to say that for

$$
\begin{aligned}
& \nabla_{\mathrm{T}} \mathrm{N}=\left(\left(\mathrm{C}_{1}-(1 / 2) \cos \beta\right) \cos _{1}\right. \\
&+\left(\mathrm{C}_{1}-(1 / 2) \cos \beta\right) \sin \mathrm{E}_{2} \\
&+((-1) / 2) \sin \beta) \mathrm{E}_{3} \\
& \nabla_{\mathrm{T}} \mathrm{N}=(1 / 2)\left(\left(2 \mathrm{C}_{1}-\cos \beta\right) \cos \mathrm{D},\left(2 \mathrm{C}_{1}-\cos \beta\right) \sin \mathrm{D},-\sin \beta\right)
\end{aligned}
$$

It is well known that Binormal vector field of a curve is $\mathrm{B}=(1 / \tau)\left(\nabla_{T} \mathrm{~N}+\kappa \mathrm{T}\right)$. Also torsion is

$$
\begin{aligned}
& \tau=\mathrm{g}_{\mathrm{Nil}_{3}}\left(\nabla_{\mathrm{T}} \mathrm{N}, \mathrm{B}\right) \\
& \tau=\mathrm{g}_{\mathrm{Nil}_{3}}\left(\nabla_{\mathrm{T}} \mathrm{N},(1 / \tau)\left(\nabla_{T} \mathrm{~N}+\kappa \mathrm{T}\right)\right) \\
& \left.\tau^{2}=\mathrm{g}_{\mathrm{Nil}_{3}}\left(\nabla_{\mathrm{T}} \mathrm{N}, \nabla_{\mathrm{T}} \mathrm{N}+\kappa \mathrm{T}\right)\right) \\
& \left.\left.\tau^{2}=\mathrm{g}_{\mathrm{Nil}_{3}}\left(\nabla_{\mathrm{T}} \mathrm{N}, \nabla_{\mathrm{T}} \mathrm{N}\right)\right)+\mathrm{g}_{\mathrm{Nil} 3}\left(\nabla_{\mathrm{T}} \mathrm{N}, \kappa \mathrm{T}\right)\right) \\
& \tau^{2}=(1 / 4)\left(\left(\left(2 \mathrm{C}_{1}-\cos \beta\right) \cos \mathrm{D}\right)^{2}+\left(\left(2 \mathrm{C}_{1}-\cos \beta\right) \sin \mathrm{D}\right)^{2}+\sin ^{2} \beta\right) \\
& \tau^{2}=(1 / 4)\left(\left(2 \mathrm{C}_{1}-\cos \beta\right)^{2}+\sin ^{2} \beta\right) \text { or } \tau^{2}=\mathrm{C}_{1}{ }^{2}-\mathrm{C}_{1} \cos \beta+(1 / 4) .
\end{aligned}
$$

\subsubsection{The Binormal Vector Field of the General Helix in Nil Space $\mathrm{Nil}_{3}$}

Theorem:

Let $\alpha: \mathrm{I} \rightarrow \mathrm{Nil}_{3}$ be a unit speed non-geodesic general helix. Then, the binormal vector field of the general helix is

$\mathrm{B}=\left(1 /\left(\mathrm{C}_{1}{ }^{2}-\mathrm{C}_{1} \cos \beta+(1 / 4)\right)^{1 / 2}\right)$

$\left(\left(\mathrm{C}_{1}-(1 / 2) \cos \beta+\sin ^{2} \beta \cos \beta-\sin ^{2} \beta \mathrm{C}_{1}\right) \cos \mathrm{DE}_{1}\right.$

$+\left(\mathrm{C}_{1}-(1 / 2) \cos \beta+\sin ^{2} \beta \cos \beta-\sin ^{2} \beta \mathrm{C}_{1}\right) \operatorname{sinDE} \mathrm{E}_{2}$

$\left.\left.+\left(\cos ^{2} \beta-\cos \beta \mathrm{C}_{1}-(1 / 2)\right) \sin \beta \mathrm{E}_{3}\right)\right]$

where we take $\mathrm{D}=\mathrm{C}_{1} \mathrm{~s}+\mathrm{C}_{2}$, where $\mathrm{C}_{1}, \mathrm{C}_{2} \in \mathrm{IR}$.

Proof:

With the Levi-Civita connection and Lie brackets can be easily computed as:

$$
\nabla_{\mathrm{T}} \mathrm{N}=(1 / 2)\left(\left(2 \mathrm{C}_{1}-\cos \beta\right) \cos \mathrm{D},\left(2 \mathrm{C}_{1}-\cos \beta\right) \sin \mathrm{D},-\sin \beta\right) .
$$

And, using the Frenet-Serret equation $\nabla_{\mathrm{T}} \mathrm{N}=-\kappa \mathrm{T}+\tau \mathrm{B}$, we have $\mathrm{B}=(1 / \tau)\left(\nabla_{\mathrm{T}} \mathrm{N}+\kappa \mathrm{T}\right)$

$$
\begin{aligned}
\mathrm{B}= & (1 / \tau)\left(\left(\left(\left(2 \mathrm{C}_{1}-\cos \beta\right) /(2 \kappa)\right)\right)\left(\cos \beta-\mathrm{C}_{1}\right)+\kappa\right) \sin \beta \cos \mathrm{DE}_{1} \\
& +\left(\left(\left(\left(2 \mathrm{C}_{1}-\cos \beta\right) /(2 \kappa)\right)\right)\left(\cos \beta-\mathrm{C}_{1}\right)+\kappa\right) \sin \beta \sin \mathrm{DE}_{2} \\
& +\left(\kappa \cos \beta-\left(\left(\sin ^{2} \beta\right) /(2 \kappa)\right)\left(\cos \beta-\mathrm{C}_{1}\right)\right) \mathrm{E}_{3} .
\end{aligned}
$$

By substituting $\kappa$ and $\tau$, we complete the proof.

Example:

Assume that $\alpha: \mathrm{I} \rightarrow \mathrm{Nil}_{3}$ be a unit speed non-geodesic general helix and its axis as parallel to the vector $E_{3}$. Also $\mathrm{g}_{\mathrm{Ni}_{3}}\left(\mathrm{~T}, \mathrm{E}_{3}\right)=\mathrm{T}_{3}=\cos (\pi / 6)$, where $\beta=(\pi / 6)$ is constant angle. Hence the explicit parametric equation of helix is

$$
\begin{aligned}
& \alpha(s)=((1 / 2) \sin s+1) \mathrm{E}_{1}+(((-1) / 2) \cos \mathrm{s}+1) \mathrm{E}_{2} \\
& \quad+((1 /(16)) \sin 2 \mathrm{~s}-(1 / 2) \sin \mathrm{s}+(((1+2 \sqrt{3}) / 4)) \mathrm{s}) \mathrm{E} 3 .
\end{aligned}
$$

Hence; the first curvature is

$$
\kappa=(1 / 2)(((\sqrt{ } 3) / 2)-1)=((\sqrt{ } 3-2) / 4)
$$

and the second curvature is

$$
\tau=(5-2 \sqrt{ } 3)^{1 / 2} / 2 .
$$




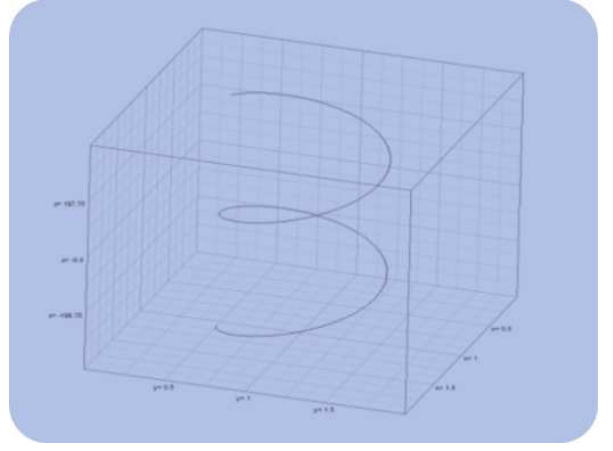

Figure 1. The figure of Helix in Example.

\section{References}

[1] Yildirim, A. and Hacisalihoglu, H.H., On BCV-Sasakian Manifold. International Mathematical Forum, 2011,Vol. 6, no. 34, $1669-1684$.

[2] Struik, D. J., Lectures on Classical Differential Geometry, Dover, New-York, 1988.

[3] Turhan, E. and Körpınar, T. " Parametric equations of general helices in the sol space $\mathrm{Sol}_{3}$," Bol. Soc. Paran. Mat., 2013, 31(1), 99-104.

[4] E. Wilson, "Isometry groups on homogeneous nilmanifolds", Geometriae Dedicata 12 (1982) 337-346.

[5] Fastenakels, J., Munteanu, M.I. and J. van der Veken,“Constant angle surfaces in the Heisenberg Group," Acta Mathematica Sinica, English Series Apr.,March 15, 2011, Vol. 27, No. 4, pp. $747-756 . ?$

[6] Kula, L. and Yayli, Y., "On slant helix and its spherical indicatrix," Applied Mathematics and Computation, 169,600-607, 2005.

[7] Lancret, M. A., Memoire sur les courbes 'a double courbure, Memoires presentes alInstitut 1(1806), 416-454.

[8] Ergüt, M., Turhan, E. and Körpınar, T., "On the Normal ruled surfaces of general helices in the Sol space $\mathrm{Sol}_{3}$ ", TWMS J. Pure Appl. Math. V. 4, N. 2, 2013, 125-130.

[9] Ekmekci, N. and Ilarslan, K., "Null general helices and submanifolds,” Bol. Soc. Mat. Mexicana, 2003, 9(2), 279-286.

[10] Izumiya, S. and Takeuchi, N. "Special curves and Ruled surfaces,". Beiträge zur Algebra und Geometrie Contributions to Algebra and Geometry, 2003, Volume 44, No. 1, 203-212.

[11] Kilıçoğlu, S., "On the Involutive B-scrolls in the Euclidean three-space $\mathrm{E}^{3}$. ., XIII ${ }^{\text {th }}$. Geometry Integrability and Quantization, Varna, Bulgaria: Sofia 2012, pp 205-214

[12] Kılı̧̧oğlu, S. and Hacısalihoglu, H. H., "On the parametric equations of the Normal and Binormal ruled surface of general helices in Nil Space $\mathrm{Nil}_{3}$," unpublished.

[13] Thurston, W., "Three-Dimensional Manifolds, Kleinian Groups and Hyperbolic Geometry.", Bull. Amer. Math. Soc., 1982, 6, 357-381.

[14] Ou, Y. and Wang, Z., "Linear biharmonic maps into Sol, Nil and Heisenberg Spaces.”, Mediterr. j. math., 2008, 5, 379-394. 